\title{
Grape-seed polyphenols inhibit AAA in mice via regulation of macrophage polarization
}

\author{
Ma $\mathrm{D}^{1}$, Shen $\mathrm{HT}^{2}$, Zhao $\mathrm{JJ}^{2}$, Chang $\mathrm{Q}^{1}$, Tian $\mathrm{LB}^{3}$, Ma $\mathrm{Y}^{3}$, $\mathrm{Li} \mathrm{L}{ }^{3}$, Tan $\mathrm{Z}^{3}$ \\ Department of Cardiology, Tangshan Workers Hospital, Tangshan, China.89167731@qq.com
}

\begin{abstract}
AIM: Abdominal aortic aneurysm (AAA) is characterized by macrophage polarization, and at present, no drug therapy is available. Although grape-seed polyphenols (GSP) showed an anti-AAA effect, the role of GSP in the phenotype shift in macrophages remains unknown.

METHOD: The main phenolic compounds in GSP were determined by LC-MS. Male C57BL/6 mice were divided into four equal groups, namely sham group, $\mathrm{CaPO}_{4}$ group, $\mathrm{CaPO}_{4}+\mathrm{GSP}$ low-dose group, and $\mathrm{CaPO}_{4}+\mathrm{GSP}$ high-dose group. GSP was administered intragastrically after $\mathrm{CaPO}_{4}$ application. Molecular expressions were histologically evaluated and analyzed by various staining assays and FACS.

RESULTS: GSP administration inhibited $\mathrm{CaPO}_{4}$-induced AAA formation, which correlated with a decrease in macrophage infiltration and retainment of vascular smooth muscle layer as compared to those in the $\mathrm{CaPO}_{4}$ group. FACS assay showed that the GSP administration dose-dependently decreased the CD54 expression (low-dose group: $11.4 \pm 2.1 \%$ and high-dose group: $4.8 \pm 1.4 \%$ vs $23.2 \pm 3.6 \% ; p<0.05$ and $p<0.01$, respectively) and increased the CD206 expression of F4/80-positive cells in GSP-administered groups as compared with that in $\mathrm{CaPO}_{4}$-injured aortas in the $\mathrm{CaPO}_{4}$ group (low-dose group: $22.4 \pm 3.3 \%$ and highdose group: $26.7 \pm 4.2 \%$ vs $8.1 \pm 1.3 \%$; $<<0.05$ and $p<0.01$, respectively).

CONCLUSIONS: GSP could be a pharmacologically potent agent in the treatment of AAA (Tab. 1, Fig. 3, Ref. 20). Text in PDF www.elis.sk

KEY WORDS: grape-seed polyphenols, abdominal aortic aneurysm, macrophage, inflammatory, polarization.
\end{abstract}

\section{Introduction}

Abdominal aortic aneurysm (AAA) refers to abdominal aortic dilation. This can be a life-threatening condition in case of its rupture. Since there are no, effective drugs for its therapy or other non-invasive treatment options, the clinical condition of AAA carries a high mortality risk (1). The main risk factors are age over 65 years, male sex, smoking history, atherosclerosis, dyslipidemia, hypertension, and family history of AAA (2). The pathogenesis of AAA is complex and multifactorial. It includes proteolytic degradation of the vessel, inflammation and immune responses, biochemical wall stress, and molecular genetics, while macrophage infiltration is a common feature (3). The modified calcium phosphate $\left(\mathrm{CaPO}_{4}\right)$ model has been widely accepted as an experimental animal model with many pathologic similarities

${ }^{1}$ School of Public Health, North China University of Science and Technology, Tangshan, China, ${ }^{2}$ Department of Neurosurgery, affiliated Hospital of North China University of Science and Technology, Tangshan, China, and ${ }^{3}$ Department of Cardiology, Tangshan Workers Hospital, Tangshan, China

Address for correspondence: Zheng Tan, Department of Cardiology, Tangshan Workers Hospital, Tangshan, China.

Acknowledgments: This work was supported by the National Natural Science Foundation of China (No. 81700416) and Tangshan city Key Scientific and Technological Project (No. 17130260a). with human AAA (4). Macrophages localized in vascular lesions suggest an inflammatory response which destroys the integrity of the vessel wall, including its endothelial and vascular smooth muscle cells (VSMCs). The latter impairment then recruits even more macrophages, thus contributing to the inflammatory cascade. The process finally leads to AAA formation and its progression $(5,6)$

The importance of macrophage phenotypic modulation in AAA has been highlighted. Under the pathophysiologic conditions of AAA, the macrophages can adopt multiple diverge functions via undergoing different states of their phenotypic polarization. Namely, the M1-type macrophages damage the contiguous tissue whereas M2-type macrophages promote the contiguous tissue repair. An imbalance in M1-M2 polarization in macrophages is often associated with inflammation or diseases, inclusive of AAA formation and its progression, whereas a proper $\mathrm{M} 1 / \mathrm{M} 2$ ratio is vital for vascular homeostasis $(7,8)$.

There is accumulating evidence indicating that in addition to their strong anti-oxidant and anti-inflammatory potential, the grape seed polyphenols (GSP) have other various biological effects (9, 10). Previous study has demonstrated that GSP plays a protective role in elastase-induced AAA in mice (11), however, the question whether GSP inhibits the macrophage inflammatory response has not yet been investigated in the context of the dynamicity of macrophage polarization. 
In light of the above facts, this study aims to evaluate the main ingredients of GSP and their anti-inflammatory effect on the regulation of macrophage polarization in a $\mathrm{CaPO}_{4}$-induced mouse AAA model.

\section{Materials and methods}

\section{Total phenolic content}

The total phenolic content (TP) was measured by Folin-Ciocalteu assay while using gallic acid as standard as described in previously protocol, albeit adapted for the use of plate reader spectrophotometer (12).

\section{The antioxidant capacity}

The antioxidant capacity (AOC) was measured by DPPH method while using trolox as standard as described in previous protocol, albeit adapted for the use of plate reader spectrophotometer (13).

\section{Polyphenols analysis by chromatography}

The main ingredients of GSP were measured by LC-MS equipment (Agilent Corporation1200 series) as previously described (14).

\section{Animal model of AAA}

Male C57BL/6 mice (Jackson Laboratory, Bar Harbor, ME, USA), 8-10 weeks old, were administered with $\mathrm{CaPO}_{4}$ to induce AAA as previously described (4). The injured abdominal arteries were harvested for analysis of RNA, morphology and histology. All experimental procedures were approved by the local Animal Care and Use Committee of North China University of Science and Technology.

\section{Experimental design}

The mice were randomized into four groups: the $\mathrm{CaPO}_{4}$ group was administered with $\mathrm{CaPO}_{4}$; Two GSP groups were administered with GSP intragastrically at a daily dose of either $400 \mathrm{mg} / \mathrm{kg}$ of body weight (low-dose GSP group) or $800 \mathrm{mg} / \mathrm{kg}$ (high-dose GSP group), starting on the first day after $\mathrm{CaPO}_{4}$ application, while the control group was administered with phosphate-buffered saline (PBS) (11).

\section{Histological analysis}

The $\mathrm{CaPO}_{4}$-injured aortas harvested from all groups of AAA mice (both, treated and not treated with GSP) were examined with the use of hematoxylin and eosin and EVG staining for their morphology, elastin degradation, macrophage infiltration and decreased VSMCs, as well as subjected to immunohistochemistry and immunofluorescent staining experiments as described previously.

\section{Flow-cytometry analysis}

Infiltrated macrophages in $\mathrm{CaPO}_{4}$-injured aortas harvested from all groups of AAA mice (both treated and not treated with GSP for 14 days as described previously, were analyzed for their phenotype by FACS (FC500 MPL Beckman).

\section{Statistical analysis}

The statistical analysis was performed using GraphPad Prism 5 software (GraphPad Software, La Jolla, CA). The data are displayed as mean \pm standard deviation (SD). The statistical significance among the groups was tested by one-way ANOVA (Bonferroni). Two-sided $\mathrm{p}$ values of $<0.05$ were considered statistically significant.

\section{Results}

\section{Chemical characterization}

Polyphenolic extract of grape seed contains high levels of total phenolic content (TP). In addition, the chromatographic analysis further revealed that the major polyphenols are gallic acid, procyanidins B1, procyanidins B2, catechins and epicatechin gallate (Tab. 1).

As shown in Figure 1A, the increase in the ratio of the maximal abdominal aortic diameter to the adjacent normal aortic diameter of aneurysm formation was significantly prevented in lowdose and high-dose GSP groups compared with the $\mathrm{CaPO}_{4}$ group ( $\mathrm{CaPO}_{4}$ group: $104.7 \%$; low-dose GSP group: $67.5 \%$; high-dose GSP group: $25.1 \%$ ). HE (Fig. 1B) and EVG (Fig. 1C) staining demonstrated pathological changes in $\mathrm{CaPO}_{4}$-induced AAA formation including the decrease in the thickness of tunica media, high infiltration of inflammatory cells into tunica adventitia, and degeneration and destruction of elastic layers within tunica media. These results suggest that GSP may have a protective effect against aneurysm formation in mice.

Grape-seed polyphenols (GSP) attenuate $\mathrm{CaPO}_{4}$-induced AAA formation.

GSP prevents VSMC reduction and reduces inflammation in $\mathrm{Ca}$ $\mathrm{PO}_{4}$-injured aortas

As shown in Figures 2A and 2B, the application of $\mathrm{CaPO}_{4}$ led to an increase in $\mathrm{F} 4 / 80$-positive staining $(641.8 \pm 98.2$ vs $26.8 \pm$ 19.2; $<<0.001)$, and a decrease in SM22 $\alpha$-positive staining (20.4 $\pm 14.0 \%$ vs $100 \pm 4.0 \%$; $<0.001)$ in the aortas of $\mathrm{CaPO}_{4}$ group as compared with those from the control group, whereas GSP administration dose-dependently and significantly decreased the macrophage infiltration (low-dose GSP group: $278.0 \pm 66.4 \mathrm{vs}$ $641.8 \pm 98.2 ; \mathrm{p}<0.05$; high-dose GSP group: $115.5 \pm 44.3 \% \mathrm{vs}$ $641.8 \pm 98.2 ; \mathrm{p}<0.01)$ as well as the loss in the thickness of tunica media (low-dose GSP group: $55.4 \pm 16.1 \%$ vs $20.4 \pm 14.0 \%$; $\mathrm{p}<0.05$; high-dose GSP group: $85.6 \pm 12.3 \%$ vs $20.4 \pm 14.0 \%$; $\mathrm{p}<0.01)$ in the $\mathrm{CaPO}_{4}$-injured aortas. The relative data are shown in Figures $2 \mathrm{~A}$ and $2 \mathrm{~B}$.

\section{GSP changes the M1/M2 transition in $\mathrm{CaPO}_{4}$-injured aortas}

Based on the FACS assay, the GSP administration dose-dependently suppressed CD54 expression of F4/80-positive cells in 
A

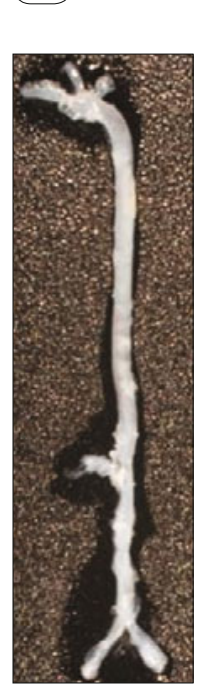

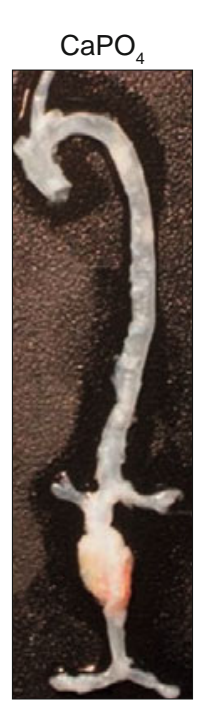

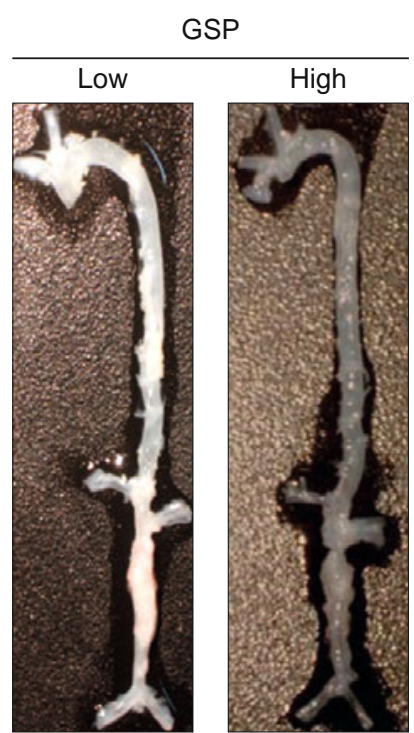

B

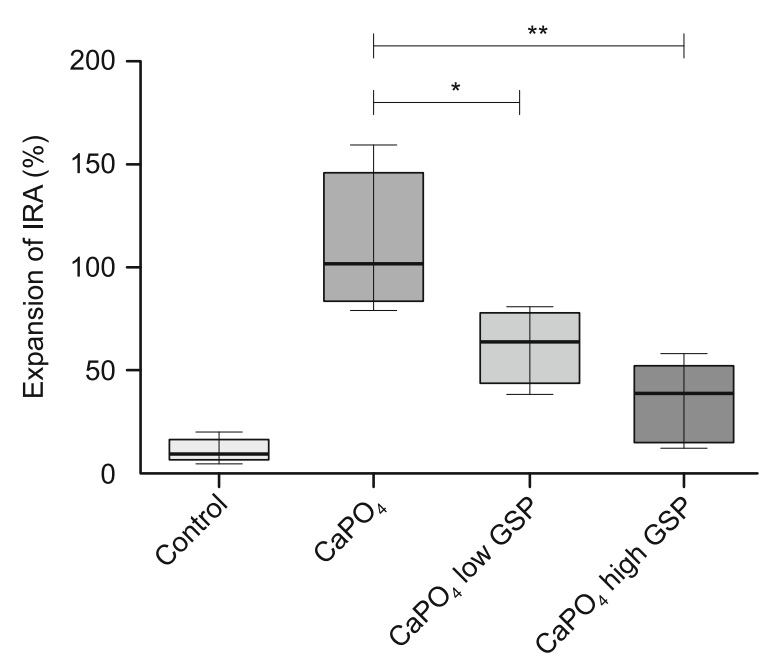

C
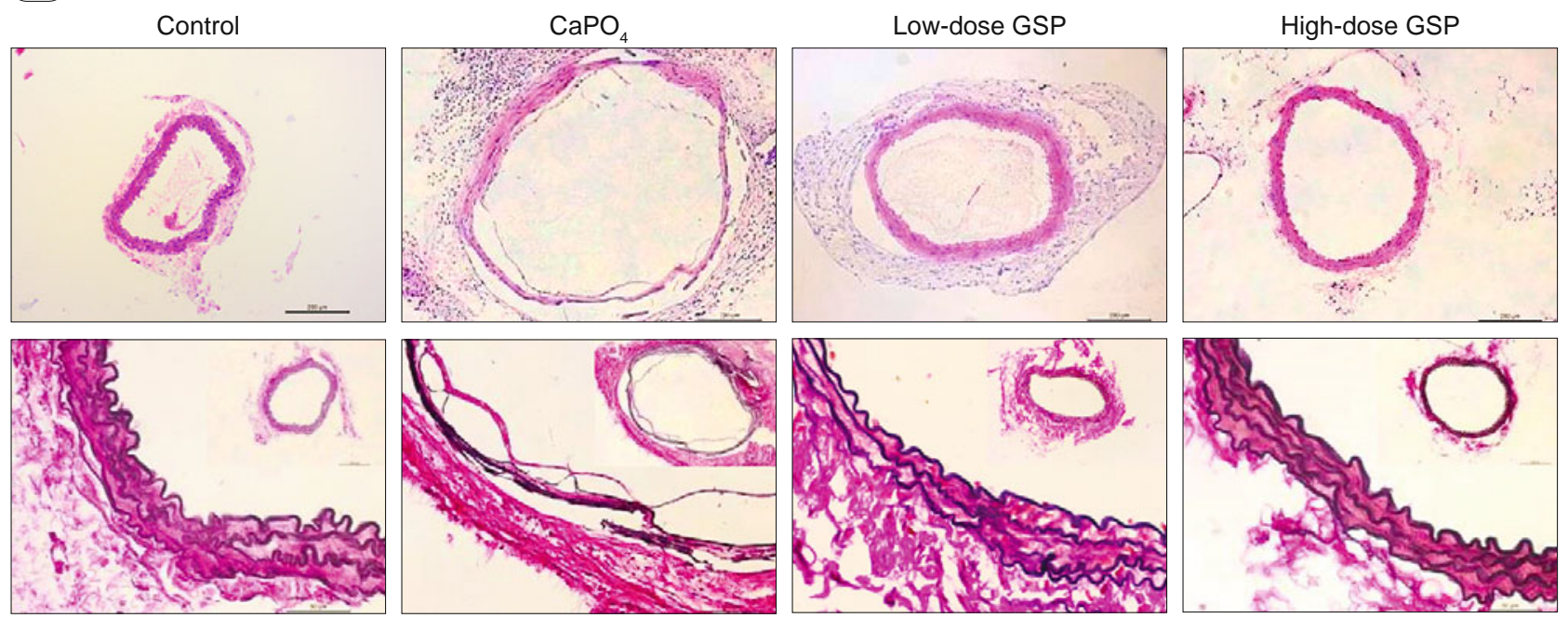

Fig. 1. GSP attenuates $\mathrm{CaPO}_{4}$-induced AAA in mice. A) Representative photographs of AAA in different groups. Scale bars $=2.5 \mathrm{~mm}$. Right: maximal percentage of abdominal aortic dilation. Data are presented as mean $\pm \mathrm{SD}, \mathbf{n}=7$ in each group. $* * * \mathbf{p}<0.001 \mathrm{vs}$ control, $* \mathbf{p}<0.05$ and $* * \mathrm{p}<0.01$ vs $\mathrm{CaPO}_{4}$ group. B) and C) $\mathrm{H} \& \mathrm{E}$ or EVG staining in 4 different groups. Scale bar $=200 \mu \mathrm{m}$ or $50 \mu \mathrm{m}$. Right: quantification of media thickness and number of elastin breaks.

low-dose GSP and high-dose GSP groups as compared with that in $\mathrm{CaPO}_{4}$-injured aortas (low-dose GSP group: $11.4 \pm 2.1 \%$, and highdose GSP group: $4.8 \pm 1.4 \%$ vs $23.2 \pm 3.6 \%$; $<0.05$ and $\mathrm{p}<0.01$, respectively). Conversely, CD206 expression of F4/80-positive cells, as a marker of M2, was significantly higher in GSP groups $(22.4 \pm 3.3 \%$ and $26.7 \pm 4.2 \%$ vs $8.1 \pm 1.3 \%$; $<0.05$ and $p<0.01$, respectively). The relative data are shown in Figures $3 \mathrm{~A}$ and $3 \mathrm{~B}$.

\section{Discussion}

In our mouse model of $\mathrm{CaPO}_{4}$-induced AAA, the GSP administration reduced abdominal aortic dilation in terms of reducing the VSMC loss and macrophage-mediated inflammation, partly via regulating the dynamic macrophage polarization. This is the first study to demonstrate the protective properties of GSP against aneurysm in $\mathrm{CaPO}_{4}$-induced AAA mice.

Numerous experiments have proven that GSP extracts from waste products from winery and juice industry possess a range of unique biological and pharmacological properties, including antioxidant, anti-mutagenic and anti-inflammatory, as well as hepatoprotective, neuroprotective, anticarcinogenic and cardioprotective properties which play a preventive role in a chemically induced injuries in a mouse model (16). Particularly, the grape seed polyphenols are marketed in the United States as commercial GSE3 dietary supplement preparations with $95 \%$ standardized procyanidins owing to their health benefits (17). 
(A)

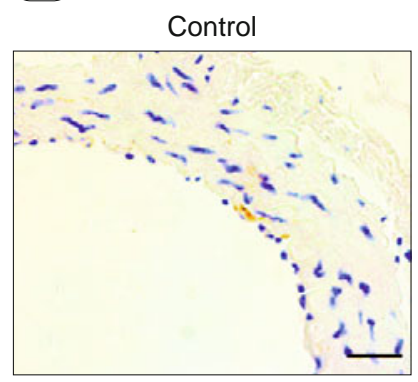

Low-dose GSP

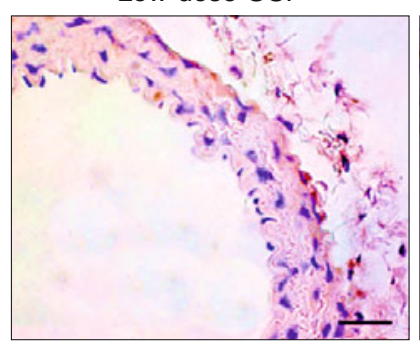

$\mathrm{CaPO}_{4}$

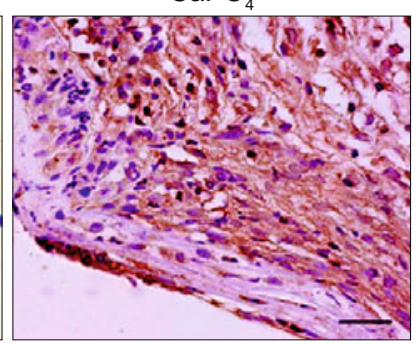

High-dose GSP

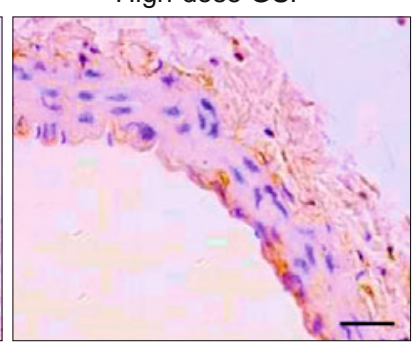

B

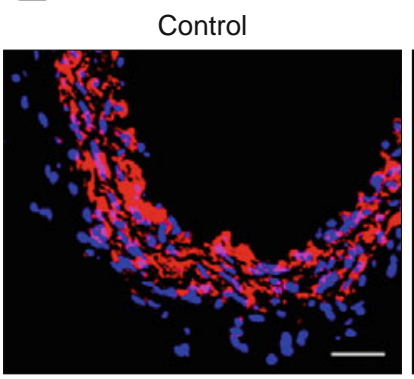

Low-dose GSP

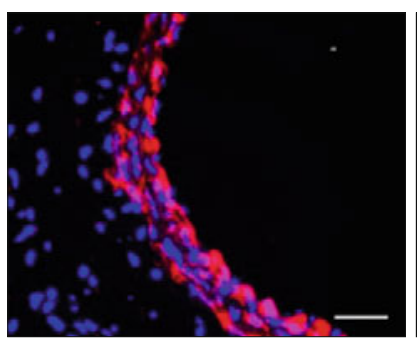

$\mathrm{CaPO}_{4}$

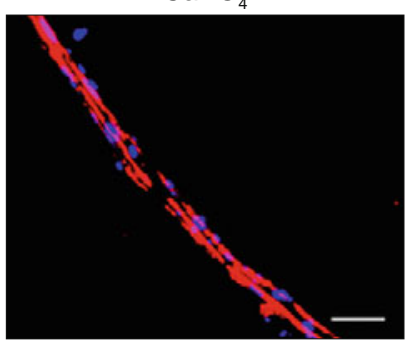

High-dose GSP

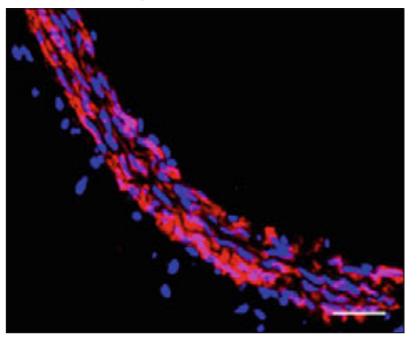

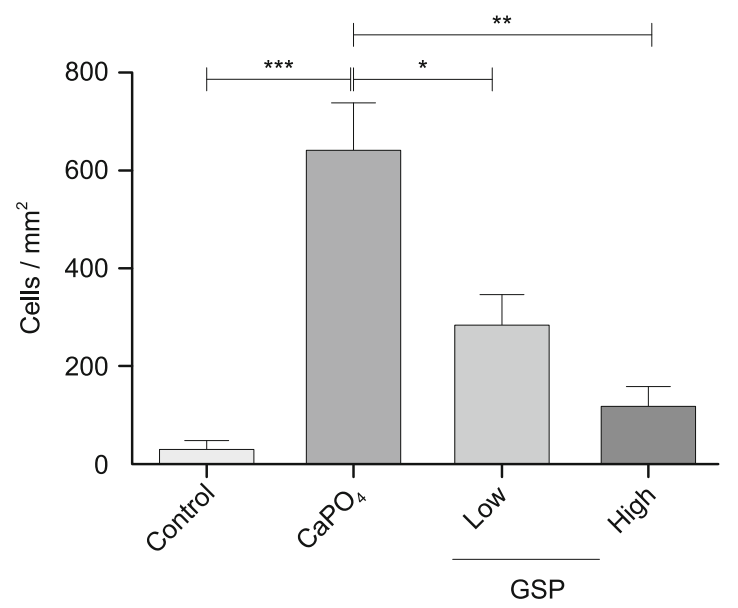

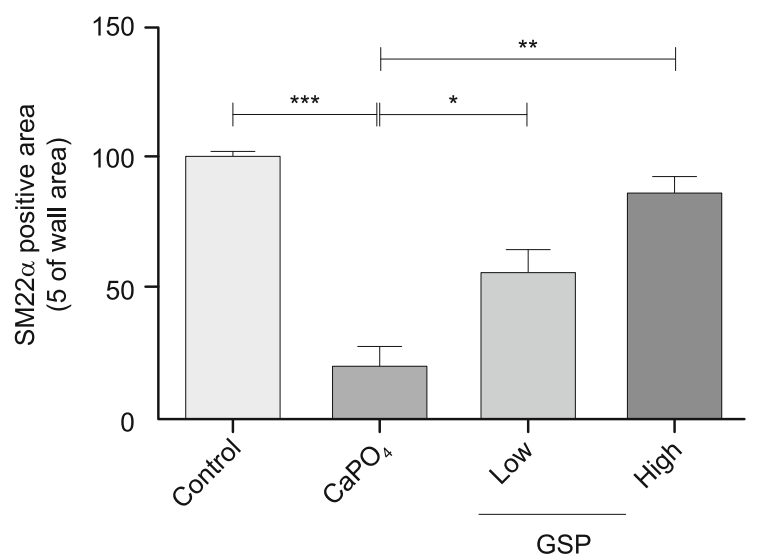

Fig. 2. GSP prevents macrophage infiltration and VSMC reduction in $\mathrm{CaPO}_{4}$-injured aortas treated or not treated with GSP. A) Immunohistochemistry staining with anti-F4/80 antibody defined the invasion of macrophages into tunica media and unica adventitia in 4 different groups. Scale bar $=40 \mu \mathrm{m}$. Down: statistics of F4/80-positive cells in aortas from 4 different groups. Data are presented as mean \pm SD, $n=3$ in each group. $* * * \mathrm{p}<0.001$ vs control, $* \mathrm{p}<0.05$ and $* * \mathrm{p}<0.01$ vs $\mathrm{CaPO}_{4}$ group. B) Immunofluorescence staining with anti-SM22 $\alpha$ antibody indicates VSMCs reduction in the aneurysmal wall across 4 different groups. Red and blue staining indicate SM22 $\alpha$ and DAPI, respectively. Scale bar $=50 \mu \mathrm{m}$. Down: quantification of SM22 $\alpha$-positive cells in tunica media in 4 different groups

Histological and immunohistochemical analyses showed that GSP administration strongly suppressed the aortic degeneration and macrophage infiltration in the aortic wall regardless of presence or absence of aneurysm. FACS analysis with $\mathrm{CaPO}_{4}$-injured aortas showed that GSP administration significantly decreased macrophage-derived CD54 expression (M1 marker) and increased macrophage-derived CD206 expression (M2 marker), which correspondingly plays a vasoprotective role via controlling the phenotype transformation of tissue-derived macrophages. Accumulating evidence (18) demonstrated that various natural products can decrease the expression of MCP- 1 and IL- $1 \beta$ molecules, thus resulting in the inhibition of macrophage invasion into the vascular wall. In this way, the macrophage infiltration and release of inflammatory mediators triggered by vascular injury may be suppressed by GSP administration, which is more likely to enhance the protective mechanism underlying the suppression of vascular inflammation and remodeling.

Wang et al (11) have demonstrated that GSP administration prevents elastase-induced AAA mouse model and inhibits the release of pro-inflammatory cytokines (MCP-1, IL-6 and IL-1 $\beta$ ) 

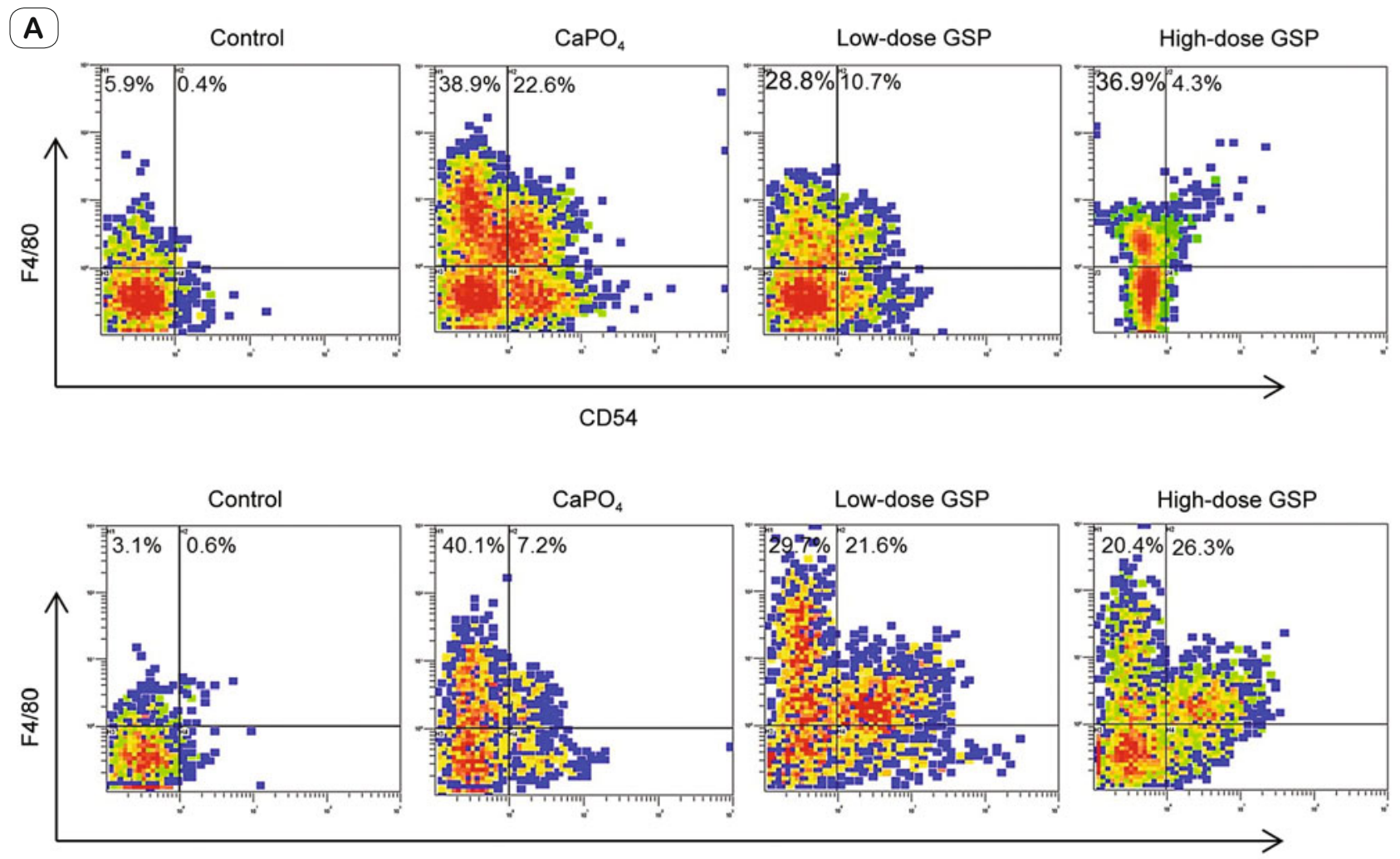

CD206

B

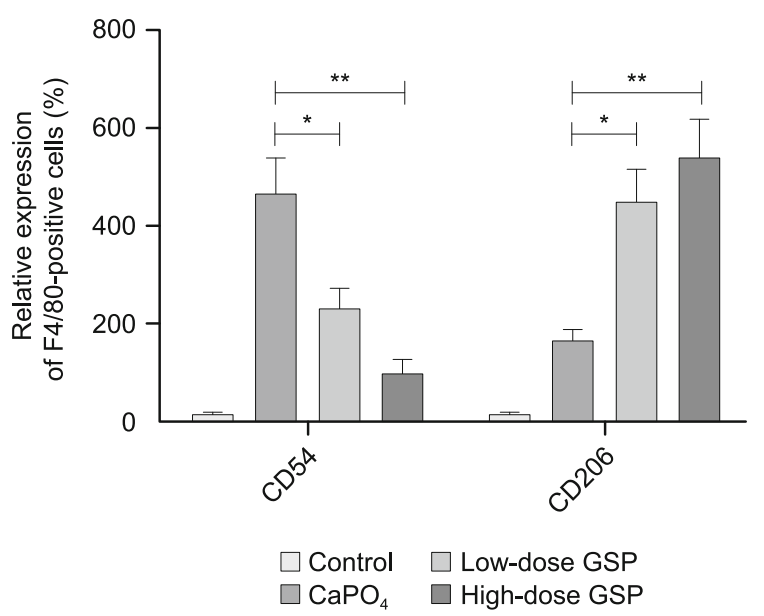

Fig. 3. FACS analysis of the dynamic macrophage polarization in aortic tissues from the control group and groups of mice exposed to $\mathrm{CaPO}_{4}$ and treated with GSP $(400 \mathrm{or} 800 \mathrm{mg} / \mathrm{kg})$. A) Representative images of FASC. B) Statistic analysis of the counts of M1 and M2 macrophages.

in TNF- $\alpha$ stimulated VSMCs. Similarly, in this study, GSP suppressed $\mathrm{CaPO}_{4}$-induced AAA formation, thus demonstrating the anti-inflammatory effects of GSP in vivo. Moreover, the GSP administration significantly prevented the accumulation of macrophages, particularly that of $\mathrm{M} 1$ macrophages and increased the ratio of $\mathrm{M} 2 / \mathrm{M} 1$ in the $\mathrm{CaPO}_{4}$-injured aortic wall. These findings are consistent with previous study (19) which demonstrated that DHA administration decreased pro-inflammatory markers in vitro and in vivo, while having a stimulatory effect on the anti-inflammatory M2 marker.

Key molecular determinants of M1-M2 polarization include members of the Notch signaling, KLF, GATA, IRF, STAT, NF-kB, HIF families and miRNAs (20). The vasoprotective effect of GSP could be partially caused by the fact that significant changes in key genes were detected, which reflects the ability of GSP to induce alterations in the dynamic macrophage polarization. Nevertheless, 
more research is needed to clearly identify the profile of these gene expressions and pathways in $\mathrm{CaPO}_{4}$-injured aortas, which is a limitation to this study.

In our present study, it was shown that GSP administration dramatically suppressed the formation of $\mathrm{CaPO}_{4}$-induced AAA and inflammation by regulating the dynamic changes in macrophage polarization. These properties of GSP contribute to its potential of opening up a new therapeutic avenue in the clinical treatment of AAA.

\section{References}

1. Wang X, Searle AK, Hohmann JD et al. Dual-targeted theranostic delivery of mirs arrests abdominal aortic aneurysm development. Mol Ther 2018; 26 (4): 1056-1065.

2. Golledge J. Abdominal aortic aneurysm: update on pathogenesis and medical treatments. Nat Rev Cardiol 2019; 16 (4): 225-242.

3. Guo DC, Papke CL, He R et al. Pathogenesis of thoracic and abdominal aortic aneurysms. Ann N Y Acad Sci 2006; 1085: 339-352.

4. Yamanouchi D, Morgan S, Stair $C$ et al. Accelerated aneurysmal dilation associated with apoptosis and inflammation in a newly developed calcium phosphate rodent abdominal aortic aneurysm model. J Vasc Surg 2012; 56 (2): 455-461.

5. Ambekar S. Inflammation and aneurysms. Neurology India 2017; 65 (4): 706-707.

6. Turner GH, Olzinski AR, Bernard RE et al. Assessment of macrophage infiltration in a murine model of abdominal aortic aneurysm. J Magn Reson Imaging 2009; 30 (2): 455-460.

7. Pope NH, Salmon M, Davis JP et al. D-series resolvins inhibit murine abdominal aortic aneurysm formation and increase $\mathrm{m} 2$ macrophage polarization. FASEB J 2016; 30 (12): 4192-4201.

8. Dale MA, Xiong W, Carson JS et al. Elastin-derived peptides promote abdominal aortic aneurysm formation by modulating $\mathrm{m} 1 / \mathrm{m} 2$ macrophage polarization. J Immunol 2016; 196 (11): 4536-4543.

9. Gupta NK, Dixit VK. Absorption enhancement of grape seed polyphenols by complexation with phosphatidyl choline. Drug Deliv 2011; 18 (5): 312-319.
10. Sakurai T, Kitadate K, Nishioka $\mathbf{H}$ et al. Oligomerized grape seed polyphenols attenuate inflammatory changes due to antioxidative properties in coculture of adipocytes and macrophages. J Nutr Biochem 2010; 21 (1): 47-54.

11. Wang C, Wang Y, Yu M et al. Grape-seed polyphenols play a protective role in elastase-induced abdominal aortic aneurysm in mice. Sci Rep 2017; 7 (1): 9402-9412.

12. Vernon LS, Rudolf O, Rosa MLR. Analysis of total phenols and other oxidation substrates and antioxidants by means of folin-ciocalteu reagent. Methods Enzymol 1999; 299 (1): 152-178.

13. Wendy BW, Cuvelier ME, Berset C. Use of a free radical method to evaluate antioxidant activity. LWT-Food Sci. Technol 1995; 28 (1): $25-30$.

14. Caro M, Sansone A, Amezaga J et al. Wine lees modulate lipid metabolism and induce fatty acid remodelling in zebrafish. Food Funct 2017; 8 (4): $1652-1659$.

15. Ma D, Zheng B, Suzuki T et al. Inhibition of KLF5-Myo9b-RhoA Pathway-Mediated Podosome Formation in Macrophages Ameliorates Abdominal Aortic Aneurysm. Circ Res 2017; 120 (5): 799-815.

16. Watson RR, Zibadi S. Skin Care Properties of Grape Seed Polyphenols, a By-Product of the Winery Industry. Bioactive Dietary Factors and Plant Extracts in Dermatology 2013; Chapter 19: 189-195.

17. Nassiri-Asl M, Hosseinzadeh H. Review of the Pharmacological Effects of Vitis vinifera (Grape) and its Bioactive Constituents: An Update: Pharmacological Effects of Grape. Phytother Res 2016; 16 (9): 1392-1403.

18. Tanaka T, Moriyama T, Kawamura $\mathrm{Y}$ et al. Puerarin Suppresses Macrophage Activation via Antioxidant Mechanisms in a $\mathrm{CaPO}_{4}-\mathrm{In}-$ duced Mouse Model of Aneurysm. J Nutr Sci Vitaminol 2016; 62 (6): 425-431.

19. Yoshihara T, Shimada K, Fukao K et al. Omega 3 polyunsaturated fatty acids suppress the development of aortic aneurysms through the inhibition of macrophage-mediated inflammation. Circ J 2015; 79 (7): $1470-1478$.

20. Wang $\mathbf{N}$, Liang $\mathbf{H}$, Zen $\mathbf{K}$. Molecular mechanisms that influence the macrophage M1-M2 polarization balance. Front Immunol 2014; 5: 614-622. 